\title{
Germanica
}

\section{Historizität und Interkulturalität im Roman Die Fische von Berlin von Eleonora Hummel}

Historicity and interculturality in the novel Die Fische von Berlin by Eleonora Hummel

Historicité et interculturalité dans la nouvelle Die Fische von Berlin de Eleonora Hummel

\section{Natalia Shchyhlevska}

\section{OpenEdition}

\section{Journals}

Édition électronique

URL : http://journals.openedition.org/germanica/2035

DOI : 10.4000/germanica.2035

ISSN : 2107-0784

\section{Éditeur}

Université de Lille

\section{Édition imprimée}

Date de publication : 31 décembre 2012

Pagination : 203-219

ISBN : 9782913857308

ISSN : 0984-2632

\section{Référence électronique}

Natalia Shchyhlevska, « Historizität und Interkulturalität im Roman Die Fische von Berlin von Eleonora Hummel », Germanica [Online], 51 | 2012, Online erschienen am: 14 Januar 2013, abgerufen am 06 Oktober 2020. URL : http://journals.openedition.org/germanica/2035 ; DOI : https://doi.org/10.4000/ germanica.2035

Ce document a été généré automatiquement le 6 octobre 2020.

(c) Tous droits réservés 


\section{Historizität und Interkulturalität im Roman Die Fische von Berlin von Eleonora Hummel}

Historicity and interculturality in the novel Die Fische von Berlin by Eleonora Hummel

Historicité et interculturalité dans la nouvelle Die Fische von Berlin de Eleonora Hummel

Natalia Shchyhlevska

"Wer fragt, muß die Antwort ertragen können." "

1 Der vorliegende Beitrag fokussiert am Beispiel des Romans Die Fische von Berlin die geschichtlich-politischen Bezugspunkte und die persönlichen Erfahrungen von Verfolgung und Emigration als zentrale Themen der russlanddeutschen Literatur. Er analysiert, wie neue, dem deutschen Leser unbekannte Schauplätze, Themen und Ereignisse in die Erzählstruktur eingebaut und dargestellt werden. In diesem Kontext kommt dem Erinnern eine besondere Bedeutung zu. Es wird aufgezeigt, dass die Literatur der Russlanddeutschen nicht nur das thematische und motivische Spektrum der deutschen Gegenwartsliteratur erweitert und Lücken im kollektiven Gedächtnis füllt, sondern einen neuen Blick auf die Geschichte der Sowjetunion ermöglicht. Darüber hinaus wird auf die Handhabung der deutschen Sprache als Literatursprache eingegangen, um $\mathrm{zu}$ veranschaulichen, wie die Erfahrung der Grenzüberschreitung versprachlicht wurde.

\section{Die Fische von Berlin im Kontext der deutschsprachigen Literatur russischer MigrantInnen}

Nationale Kulturen und insbesondere Literaturen Westeuropas verdanken eine explizite Entwicklung und Erweiterung ihrer Inhalte, Fragen und Motive sowie ihre sprachliche und ästhetische Gestaltung den Einwanderungsprozessen, die seit Mitte des 
20. Jahrhunderts andauern. In den letzten Jahren erlebt der deutsche Buchmarkt eine Explosion von Neuerscheinungen aus der Feder der MigrantInnen. Die Liste der mit dem Adelbert-von-Chamisso-Preis ausgezeichneten deutschsprachigen AutorInnen nichtdeutscher Sprachherkunft zeugt von der großen Vielfalt der Interkulturellen Literatur. Das Phänomen „The New Nabokovs“, wie in den USA die Literatur von David Bezmozgis, Olga Grushin, Gary Shteyngart und Lara Vapnyar bezeichnet wird, ist in Deutschland nicht nur längst angekommen, sondern hat sich in seiner für die Geschichte Deutschlands spezifischen Breite manifestiert. Die deutschsprachige Literatur russischer MigrantInnen konstituiert sich aus der jüdisch-russisch-deutschen Literatur (Lena Gorelik, Olga Grjasnowa, Wladimir Kaminer, Olga Martynova und die in Österreich lebenden Julya Rabinowich und Vladimir Vertlib) ${ }^{2}$ und der deutschsprachigen Literatur der Russlanddeutschen (Nelly Däs, Eleonora Hummel, Wendelin Mangold, Alexander Reiser, Nelly Wacker und Ilona Walger). Während die meisten der jüdisch-russisch-deutschen AutorInnen in den 90er Jahren als Kontingentflüchtlinge die Sowjetunion verließen und als Generation P (Perestrojka) bezeichnet werden, durften die Russlanddeutschen abhängig von der politischen Situation in der ehemaligen UdSSR in vorgegebenen „Wellen“ nach Deutschland ausreisen ${ }^{3}$. Mittlerweile haben diese AutorInnen mehrere Bücher vorgelegt und sind ein nicht wegzudenkender Teil der deutschsprachigen Gegenwartsliteratur geworden. Dabei setzen sie neue thematische und inhaltliche Schwerpunkte und prägen die deutsche Gegenwartsliteratur auf der sprachlich-stilistischen Ebene. Dies soll exemplarisch anhand des Romans Die Fische von Berlin von Eleonora Hummel aufgezeigt werden.

3 Der 2005 erschienene Debütroman Die Fische von Berlin ${ }^{4}$ von Eleonora Hummel bestätigt die Beobachtung, dass der Erstling fast jedes Autors, der in der neuen Sprache schreibt, sehr starke autobiografische Züge aufweist. Chiellino erklärt dies durch das Streben nach Kontinuität: „Der interkulturelle Zwang zur Autobiographie ergibt sich aus der existentiellen Notwendigkeit, das Vorleben des lyrischen Ichs, das in einer anderen Sprache stattgefunden hat, als Bestandteil des weiteren Lebens in der neuen Sprache zu integrieren. ${ }^{.5}$

Die Frage, inwieweit Deutsch für die 1970 in Kasachstan geborene Eleonora Hummel, die laut eigener Auskunft bereits mit zehn Jahren auf Russisch ihren ersten Roman zu schreiben begann ${ }^{6}$, eine neue Sprache war, bedarf einer kurzen Erläuterung. Im Roman beschreibt sie, dass die Großelterngeneration ein Deutsch gesprochen hat, das „an Konserven, die $\mathrm{zu}$ lange im Keller gestanden und einen seltsamen Geschmack angenommen hatten“ (S. 158) erinnerte. Wie war dann das „Deitsch“, das die Enkel sprachen, wenn sie es überhaupt gelernt hatten? Irma, die ältere Schwester der IchErzählerin, war bereit zur Assimilation und weigerte sich, zusammen mit der Familie nach Deutschland auszureisen: „Ich weiß nicht, wie es dir geht, aber ich kann es nicht mehr hören, dieses Gerede von Heimat und Vaterland! Von der Sprache, die unsere Kinder nicht mehr lernen werden. Was soll ich mit einer Sprache, die meine Urgroßmutter irgendwann irgendwo gesprochen hat? Ich lebe jetzt und hier, und hier spricht man anders." (S. 34).

5 Um sich an ,jetzt“ und „hier“ maximal anzupassen, heiratete Irma ohne große Liebe nur, um ihren deutschen Namen „Schmidt“ möglichst schnell loszuwerden (S. 111). Die zwölfjährige Ich-Erzählerin wird dagegen von ihrem Umfeld ständig an die deutsche Zugehörigkeit erinnert: für ihre Spielkameradinnen war sie Faschistin, ihre 
Russischlehrerin lobte ihre „Leistungen, ,die um so bemerkenswerter sind, als Russisch nicht deine Muttersprache ist'. [...] Genauso gut hätte sie sagen können, ich solle mich vor der Klasse ausziehen." (S. 178).

Die Autorin stattet die Ich-Erzählerin mit zwei Sprachen aus: das Russische, die Sprache des Umfeldes, trifft auf das Deutsche, die Sprache der Erzählung. Wie schlägt sich dies in der Sprache des Romans nieder, wie wird das Russische im Deutschen sichtbar, in wieweit beeinflusst dies die thematische und inhaltliche Ebene des Romans?

Der Roman ist eine Familiensaga mit einem obligaten Familiengeheimnis, das die IchErzählerin lüftet, indem sie den Großvater zum Sprechen bringt. Ein kleines Taschenmesser unter dem Kopfkissen des Großvaters, das angeblich „gut gegen seine Krankheit“" (S. 18) war, und ein altes Foto, das „fünf Männer, die barfuß in hochgekrempelten Hosen auf einer Waldlichtung saßen“ (S. 16) zeigt, mit der Notiz „Igarka, 1956“ wecken die Neugier der Ich-Erzählerin. Da sie weder von der Schwester noch von der Großmutter und den Eltern Antworten bekommt, welche Krankheit denn der Großvater habe und wo Igarka liege, bleibt ihr nichts anderes als den Großvater selbst zu fragen. Das im Roman für die Entwicklung der Handlung verwendete Modell des Gesprächs zwischen der Ich-Erzählerin und dem Großvater, insbesondere die Tatsache, dass aus der Großelterngeneration ausgerechnet der Großvater von der Vergangenheit erzählt, scheint eine seltene Besonderheit, wenn nicht schlechthin eine Ausnahme in der deutschsprachigen Literatur russischer MigrantInnen darzustellen. Denn für die meisten Werke, insbesondere für die der jüdisch-russisch-deutschen AutorInnen ist das Ausbleiben der Großväter typisch. Die Großmütter sind zwar am Leben, aber sie sind dement $^{7}$ oder schweigen über die Vergangenheit ${ }^{8}$, so dass die Generation der Enkelkinder bezüglich der Vergangenheit ihrer Familie im Dunkeln tappt und das Familiengeheimnis kaum geklärt werden kann. Die Umgangsweise der Großmütter mit ihrer Geschichte kann für das nicht gelebte, verdrängte und fremd gewordene Judentum der Großelterngeneration zu Sowjetzeiten stehen'. In diesem Zusammenhang käme dem Roman Die Fische von Berlin im Hinblick auf die Vergangenheit des Großvaters und die im Roman exponierten Themen - stalinistische Säuberungen 1937 sowie Verbannung und Straflager - ein Sonderstatus zu. Die offenbarung, jedoch nicht der leibliche Großvater zu sein, löst diese Besonderheit auf und verbindet dieses Buch mit dem Roman „Das französische Testament" von Andreï Makine $^{10}$, einem französischen Schriftstellers russischer Herkunft.

\section{Historizität}

Der Begriff „Historizität“ impliziert in diesem Beitrag, dass der Roman sich auf „individuelle, unwiederholbare Ereignisse "11 und Fakten bezieht, diese in die Erzählung einfließen und so die Protagonisten des Romans auf dem Hintergrund des faktischen Geschehens erscheinen lässt. Die wichtigsten, im Roman ausführlich thematisierten historischen Geschehnisse sind: (1) die Großen Säuberungen (rus.: чистки), (2) die Situation der Deutschstämmigen in den von NS-Truppen besetzten Ostgebieten und die Evakuierung der „Volksdeutschen“ sowie (3) die (massenhafte) Inkriminierung nach $\$ 58$ des Strafgesetzbuches der UdSSR und die Verurteilungen zur Zwangsarbeit in Sibirien. Im Folgenden werden diese „Geschichtstransformationen“, die „Umwandlung und Umschreibung sowohl von historischen Ereignissen als auch von 
Vergangenheitskonzeptionen in Literatur und/oder durch literarische Mittel ${ }^{{ }^{112}}$, im Roman Die Fische von Berlin aufgezeigt.

9 „Was vergangen ist, soll man nicht wieder aufrühren.“ (S. 52). Mit diesem Satz wehrte sich der Großvater anfangs gegen die Fragen seiner Enkelin. Da sie hartnäckig bleibt und der alte Mann wohl den Verfall seiner Lebenskraft spürt, entscheidet er sich, seine Vergangenheit Stück für Stück der Enkelin zu offenbaren. Eleonora Hummel knüpft an die Tradition der mündlichen Überlieferung an und erzählt die Lebensgeschichte des Großvaters aus der Perspektive und Wahrnehmung eines Kindes, dem die Geschichte vom Großvater selbst berichtet wurde. Dieses Modell ist deshalb bezeichnend, weil es die Aneignung des historisch-kulturellen Gedächtnisses demonstriert: Der Großvater ist der Wissende, der Sprecher, und weiht die Enkelin trotz der Überzeugung der Mutter „Ich wollte es niemals wissen. Wozu auch? Dieses Wissen ist unnütz.“ (S. 39) in seine Vergangenheit ein, wodurch das etablierte Verdrängungsprogramm im Umgang mit der Vergangenheit unterbrochen wird.

Das Dorf hieß Timofejewka, im Gebiet Stalino, aber wir nannten es nur die Nummer drei, weil es noch zwei andere gab mit demselben Namen. Dort bin ich aufgewachsen. [...] Es begann nicht erst 1937, und es war danach nicht plötzlich zu Ende, das, was man die Großen Säuberungen nannte. Vielleicht wurde in diesem Jahr am gründlichsten gesäubert. Die Bänke vor den Häusern blieben lange leer.

11 Wenn ich mich heute frage, warum ich jenes Jahr überlebt habe, fällt mir als erstes Mutter ein. Abends jagte sie uns aus dem Haus: Geht! Bevor sie kommen! Sie kamen in der Zeit zwischen Sonnenuntergang und Sonnenaufgang, in einem Schwarzen Raben, der nicht schwarz war. (S. 83)

Das Gebiet Stalino sucht man heute vergeblich auf der Landkarte. Es lag nicht in Sibirien, nicht in Kasachstan und nicht im Kaukasus. Von 1924 bis 1961 hieß das ostukrainische Gebiet Donezk zu Ehren des großen Diktators Stalino. Die drei gleichnamigen deutschen Dörfer im Gebiet Stalino gründeten vermutlich die Nachkommen der deutschen Siedler aus Westpreußen, die 1787/88 nach Russland kamen und Land gegenüber der Insel Chortitza, in der Nähe der heutigen Stadt Saporoshje (damals Alexandrowsk) bekamen ${ }^{13}$. Ob ihre Enkel und Urenkel die Siedlung Chortitza im Zuge des Bürgerkrieges, der nach der kommunistischen Machtübernahme 1917 entstand und bis 1921 dauerte, verließen, um sich vor Angriffen verschiedener Banden auf die wohlhabende deutsche Kolonie zu retten, oder erst 1926 beim Bau des Dneprogres Staudamms ihre Orte verlassen mussten, geht aus dem Roman nicht hervor. Im Satz „Wenn wir unter uns waren, nannte er sie die Bande.“ (S. 25), manifestiert sich aber nicht nur die Aversion des Vaters der Ich-Erzählerin gegenüber der sowjetischen Behörde, die seine Ausreise nach Deutschland seit 20 Jahren nicht erlaubte, sondern schwingt in meinem Verständnis eine weitere Konnotation mit: Im kulturhistorischen Gedächtnis des Vaters müsste das Wort „Bande“ mit der Erinnerung an Nestor Machnos Bandenarmee, die sich durch ihre Gewalt gegenüber deutschen Siedlern besonders auszeichnete ${ }^{14}$, besetzt werden. Die Kursivierung des Wortes „Bande“ im Roman kann als Indiz für die notwendige historische Dechiffrierung dieses Begriffes aufgefasst werden.

Der Satzanfang „Es begann nicht erst 1937 [...]“ exponiert die Aufhebung der vermeintlichen auf 1937 datierten Zäsur, lässt aber aus, was vor 1937 war. Die Entkulakisierung in den 20 ern und Kollektivierung 1930 forderte das Leben von Millionen Menschen ${ }^{15}$. Die Kollektivierung war gegen Bauern gerichtet, die sich der 
staatlichen Macht nicht bedingungslos unterordneten. „Angebliche und tatsächliche Kulaken werden von ihren Wirtschaften vertrieben, umgesiedelt und physisch "liquidiert", ihr Eigentum [...] wird als Anteil der Landarbeiter und der Dorfarmut in die neu entstehenden Kollektivwirtschaften, die Kolchosen, eingebracht. ${ }^{\text {“16 }}$ Die letzte Ziege und der letzte Sack Getreide wurden weggenommen, Frauen wurden erschossen oder für Jahrzehnte nach Sibirien verbannt, weil sie für ihre verhungernden Kinder auf dem ehemals eigenen Feld ein paar Weizenkörner auflasen. Die dem Hungertod oder der Erschießung entkommenen „Kulaken“ wurden nach Sibirien deportiert, wo sie in Gefängnissen und Arbeitslagern Wälder roden, Straßen und Kanäle bauen, in Gold- und Uranminen schuften mussten. „Belomor. Lange wußte ich nicht, wofür dieses Wort steht. Der Ostsee-Weißmeer-Kanal. Erbaut von Volksfeinden, an die nichts erinnert außer einer Zigarettenmarke.“ (S. 221). Der Belomorsko-Baltijskij-Kanal war eines der vielen von Volksfeinden ausgeführten Großbauprojekte des Stalinismus.

14 „Es begann nicht erst 1937, und es war danach nicht plötzlich zu Ende [...]“. Die mörderische Ausbeutung der Volksfeinde war selbst nach dem Zweiten Weltkrieg nicht zu Ende. In Kriwoi Rog und Gorlowka, nur wenige Kilometer von Timofejewka, dem Dorf des Großvaters der Ich-Erzählerin, wurde Oskar Pastior im Januar 1945 von den Sowjets als Angehöriger einer deutschsprachigen Minderheit in Rumänien deportiert und musste bis November 1949 „Reparationsarbeiten im Namen der kollektiven Schuld“ (Herta Müller) ${ }^{17}$ in einer Kokerei leisten: ein Häftling in einem Arbeitslager wie Herta Müllers Mutter, wie Tausende Deutsche aus Rumänien, wie Hunderte überlebende Juden in der Ukraine, wie die von der Roten Armee befreiten und zurückgekehrten Ostarbeiter und Gefangenen, wie zuvor die deutschen Siedler und Gegner des Kommunismus. Dass sie nach dem Krieg Donbass, das Zentrum des ukrainischen Bergbaus und der Metallurgie, aufbauten, dass die Arbeitskraft der Häftlinge - wer konnte es in der Willkür des Systems nicht werden - in unzähligen Lagern ausgenutzt wurde, dass Volksfeinde Dutzende Belomorsko-Baltijskij-Kanäle und Magnitogorsks erbauten, wurde lange verheimlicht und tabuisiert. Mit dem Roman Die Fische von Berlin deckt Eleonora Hummel diesen „weißen“ Fleck auf und schreibt ihn in das kulturhistorische Gedächtnis der deutschen Literatur ein.

Der Großvater der Ich-Erzählerin wurde wie sein Vater und Bruder während der Säuberung im Sommer 1937 abgeholt, als er kurz zu Hause auftauchte, um die Wäsche zu wechseln. Der Brief eines anderen nach Kanada ausgewanderten Bruders mit der Nachricht über die Geburt des Kindes und die geplante Eröffnung einer Konservenfabrik wurde als „Beziehungen zu einer feindlich gesinnten ausländischen Macht“" (S. 93) gedeutet. Der Großvater und sein Bruder wurden nach einigen Verhören freigelassen, was einem Wunder glich. Sein Vater kehrte aber nie wieder zurück. Erst Jahre später erfuhr die Familie, dass er bereits 1937 in Untersuchungshaft gestorben war.

16 Ich weiß, seine Knochen liegen irgendwo in einem ,Brudergrab'; seine Todesurkunden lagern in irgendeinem Archiv; die eine, die einsehbar ist, lautet auf Tod durch Herzversagen; die andere, die geheim ist, lautet auf Tod durch Erschießen; und beide tragen ihre Wahrheit in sich.

17 Niemand wird mir den Ort dieses Massengrabes nennen können (S. 100).

18 Kaum waren die Großen Säuberungen $\mathrm{zu}$ Ende, brach der Krieg aus. Für Deutschstämmige, die sowjetische Bürger waren, stellte der Krieg zwischen der Sowjetunion und dem Nazideutschland eine besondere Verwicklung dar. In 
geschlossenen Wagen mussten Männer und Frauen in einer ihnen unbekannten Richtung das Dorf verlassen, wurden wie „Faschistenpack“ (S.133) behandelt und mussten eine Befestigungsanlage um Stalino, wie sie später erfuhren, bauen. Die Industriestadt wurde bombardiert und mit ihr auch die Schützengräben: „Deutsche Bomben, die einen Bogen um ihre Landsleute hätten machen sollen, hatten unsere Zelte hinweggefegt.“ (S. 149).

Das nach der Bombardierung entstandene Chaos nutzte der Großvater aus, um zusammen mit seiner Schwester in ihr Heimatdorf zu fliehen. Das verlassene Dorf wurde bald darauf von NS-Truppen besetzt, die erwarteten, als „Befreier“ mit Brot und Salz empfangen zu werden (Vgl. S. 161). Von ihnen wurde der Großvater als Starosta des Dorfes eingesetzt. Zwei Jahre später, im Januar 1945, als sowjetische Truppen die Weichsel-Oder-Operation ${ }^{18}$ starteten, wurde die deutsche Bevölkerung unter chaotischen Umständen evakuiert: „Gerüchte kamen auf, aus denen Befehle wurden. Aus den deutschen Dörfern westlich des Dnjepr sollten Trecks mit Zivilisten in den Warthegau evakuiert werden. Zur Entlausung. Registrierung. Kategorisierung. Einbürgerung. Mobilisierung der Männer zum Wehrdienst. Und ab an die Ostfront. (S. 171).“ Das Ende des Krieges erlebte der Großvater als Zwangsarbeiter in der Nähe von Berlin. Er schmiedete Pläne, die Bauerntochter Thea zu heiraten, aber „bald kamen Gerüchte über Zwangsrepatriierungen auf. Die Sowjetorgane machten Jagd auf Sowjetbürger, ehemalige/gewesene oder auch nicht, spürten sie auf, steckten sie von der Straße weg in Lager hinter Stacheldraht, die Sammellager für Heimkehrer hießen. Die Abgesandten der Organe versprachen ihnen ein neues schönes Leben im Siegerland, wo es bald alles im Überfluß geben würde. Es sei doch die Heimat.“ (S. 174).

Zwangsarbeiter und vor allem Kriegsgefangene mussten fürchten, vom Militärtribunal zu Vaterlandsverrätern erklärt $\mathrm{zu}$ werden. Diesem Schicksal entkam auch der Großvater der Ich-Erzählerin nicht: „Der Satz, der meine Zukunft ausmachte, bestand aus drei Wörtern: Fünfundzwanzig Jahre Zwangsarbeit.“ (S. 175). „Hinter dem Wort Zwangsarbeit kam noch in Straflagern des äußersten Nordens Sibiriens." (S. 185). Das, was der Großvater seiner Enkelin berichtete, kam in keinem sowjetischen Geschichtsbuch vor. Dabei war es das Schicksal Tausender Menschen, die nach wochenlanger Fahrt in Viehwaggons das ewige Eis erreichten, um wie Tausende Volksfeinde zuvor, wie der Mann seiner Schwester Berta, unter Temperaturen, die bis -50 Grad fielen, die von Geologen entdeckten Gold-, Silber-, Platin-, Nickel- und Kobaltvorkommen abzubauen, Straßen und Brücken zu bauen und neue Siedlungen aus dem Polarboden zu stampfen. „Dort bekam ich ein neues Zuhause, Heimatersatz für einen Vaterlandsverräter - das Norillag.“ (S. 187).

21 Das Norillag wurde als „Norilskij Umerziehungsarbeitslager“ auf die Anordnung des Zentralkomitees der UdSSR vom 23. Juni 1935 gegründet und am 1. September 1956 geschlossen. Es bestand aus über 30 Lagerfilialen in Norilsk, Dudinka, Igarka, Krasnojarsk, auf der Insel Tajmyr sowie in den Dörfern Kurejka, Atamanowo und Schuschenskoje ${ }^{19}$. Der Historiker und Schriftsteller Lew Gumiljew, der Sohn von Anna Achmatowa, wurde $1938 \mathrm{zu} 5$ Jahren im Norillag verurteilt. 1938-1940 schrieb Achmatowa das Poem Requiem, eines der ersten literarischen „Klagelieder“ gegen den Stalin-Terror, das erst 1987 in der Sowjetunion veröffentlicht werden konnte. Bis dahin wurde versucht, das Norillag zu verschweigen. Die Kindergeneration, die als Kinder der Volksfeinde verachtet und in der freien Wahl der Ausbildung oder des Studienfaches behindert wurde, wollte die schmerzende Wunde nicht ständig aufs Neue aufreißen. 
Der „nervöse Magen“ des Vaters (S. 25), dieses unsichtbare körperliche Leiden, ist in meiner Lesart eine Metapher für die tabuisierte traumatische Vergangenheit der Elterngeneration. Diesen Schmerz gaben sie an ihre Kinder weiter: „Ich war in dem Bewußtsein aufgewachsen, allein durch meine Existenz eine Mitschuld an seinen körperlichen Leiden zu tragen." (S. 25).

Die Fische von Berlin kann durchaus als historisch-dokumentarischer Roman bezeichnet werden, denn die darin beschriebenen Ereignisse lassen sich mit einem Handbuch zur Geschichte der Sowjetunion bis ins Detail verifizieren. Daten und Ortsnamen - Dudinka (S. 187), Igarka (S. 17, 206), Stalino - sowie der Aufstand Ende Mai 1953 im Gorlag und die Schließung des Norillags 1956 sind reale historische Fakten. Selbst die Rechtsgrundlage, den Paragraphen 58, nach dem der Großvater verurteilt wurde, zitiert die Autorin, indem sie Alois Metzger, einen guten Freund des Großvaters, fragen lässt:

„Und sag, wie steht's mit dir? Laß mich raten: 58 Punkt eins, drei oder vier?

Fünfundzwanzig Jahre, sagte ich. Alois pfiff durch die Zähne. Es bedurfte keiner weiteren Erklärungen, er hatte begriffen. Fünfundzwanzig Jahre bedeutete knapp an der Todesstrafe vorbei, mit der Ungewißheit, ob man sie nicht doch noch bekam, langsam und in Raten." (S. 194) ${ }^{20}$.

Der Paragraph 58 des Strafgesetzbuches wurde am 25 Februar 1927 durch das Zentrale Exekutivkomitee der UdSSR verabschiedet und bestand aus 18 (!) Absätzen, die Staatsverbrechen und konterrevolutionäre Tätigkeit ausführlich definierten.

Paragraph 58 - den bekamen die Politischen: die vermeintlichen Spione, deren Schuld darin bestand, einen ausländisch klingenden Namen $\mathrm{zu}$ tragen, die Konterrevolutionäre, die nicht wußten, wie man dieses Wort buchstabiert, die heimatlosen Vaterlandsverräter, die Schädlinge, die einmal eine Handvoll Korn vom staatlichen Feld für die aufgeblähten Bäuche ihrer Kinder mitgenommen hatten. Paragraph 58 machte die Gedanken strafbar, machte den Verdacht strafbar, machte die Absicht strafbar. Für den mit Paragraph 58 bewaffneten Richter war die Stirn des Angeklagten eine Wand aus Glas. Niemandem gelang es, dahinter etwas zu verbergen, was nicht zu seinen Lasten ausgelegt werden konnte. (S. 192-193).

\section{Interkulturalität}

„Das, was man die Großen Säuberungen nannte“ (S. 83), ließ das ganze Land in Angst und Terror untergehen ${ }^{21}$. Das Schlimmste war, dass man wusste, dass es keiner Begründung bedurfte, um abgeholt, gefoltert und bestraft zu werden. Man durfte niemandem trauen. Die Willkür steigerte die Angst:

Die Angst um die eigene Haut grenzte an Wahnsinn. Sie machte jedes Gesicht austauschbar und häßlich. Es gab keine jungen Gesichter mehr im Dorf, wir trugen alle denselben Ausdruck. Ich tastete die Angst mit den Händen, wenn ich mir über die geschlossenen Augen fuhr. Sie fühlte sich an wie eine Maske, die zu fest an der Haut klebte, um sie herunterreißen zu können. Ich wollte nicht in mein Spiegelbild sehen. Mutter hatte unseren einzigen Spiegel umgedreht. So blieb er stehen, mit dem Gesicht zur Wand, bis zum Ende. (S. 84).

Der umgedrehte Spiegel in diesem Zitat ist ein Symbol: Noch heute ist es in der Ukraine und in Russland üblich, die Spiegel im Hause des Verstorbenen mit dunklen Stoffen zu 
verhängen oder umzudrehen. Der umgedrehte Spiegel im Haus des Großvaters steht für den nahen Tod, dem man sich ausgeliefert, ja geweiht fühlt. Ein weiteres Sinnbild des Todes birgt sich in der volksmündlichen Bezeichnung des Fahrzeuges, mit dem die Volksfeinde abgeholt wurden: Tschjornyj woron, „ein geschlossener Lastwagen für Gütertransporte, mit schmalen Bänken an den Längsseiten“ (S. 92). „Sie kamen in der Zeit zwischen Sonnenuntergang und Sonnenaufgang, in einem Schwarzen Raben, der nicht schwarz war." (S. 83) „Wenn der Schwarze Rabe mit dem Volksfeind wieder davonfuhr, zeichneten sich im Türrahmen die Schatten von dessen Frau und Kindern ab.“ (S. 85). Kaum jemand schlief in der Nacht. Die Angst raubte den Schlaf. Angst zu säen gehörte zum Programm, deshalb hörte und sah man nachts oft den Schwarzen Raben vor dem eigenen Haus: „Manchmal schlitterte ein Auto langsam heran, näherte sich ihrem Haus und machte nach kurzem Zögern kehrt.“ (Makine, S. 123). Ob die Bezeichnung des Unheil bringenden Fahrzeuges eindeutig auf das Volkslied der russischen Donkosaken oder ein verwandtes ukrainisches Volkslied ${ }^{22}$ zurückzuführen ist, oder sich aus dem in verschiedenen Kulturen verbreiteten Aberglauben, der Tod erscheine in Gestalt eines Raben ${ }^{23}$, speist, ändert nichts an seiner eindeutigen Metaphorik: der Schwarze Rabe war ein Todesbote in der „gutgeölten[n] Maschine der Repression“. (Makine, S. 125). Später wurden auch Lieferwagen mit der Aufschrift „Konserven“ oder „Brot“ für die Transporte der Volksfeinde verwendet - die weißen Raben. Man war erfinderisch „im Kampf gegen Ungeziefer, Parasiten, Abschaum. Strichlisten abarbeiten, Quoten erfüllen, Erschießungen anordnen, Lager zur Umerziehung von Kakerlaken errichten." (S. 86). Die Methoden waren einfach und effizient: „Tags kamen sie in die Häuser, legten ein Blatt Papier vor den Hausherrn und sagten: Gib uns die Namen von zehn Volksfeinden, und wenn es nur neun sind, dann bist der zehnte du. Niemand wollte der zehnte sein. Sie bekamen die Zettel zurück, mit zehn, zwölf, zwanzig Namen, und nachts arbeiteten sie die Liste ab.“ (S. 85). 


\section{Erlebnisse und Erfahrungen weitergibt. Der Roman Die Fische von Berlin changiert} zwischen dem Tabuisieren der Vergangenheit und dem Wunsch der zwölfjährigen Alina, die Geschichte des Großvaters zu erfahren. Es ist nicht nur die kindliche Neugier zu wissen, warum der Großvater einer Freundin im Lebensmittelladen außer der Reihe bedient wird und sie über seine Heldentaten im Großen Vaterländischen Krieg ein Referat in der Schule halten kann, während Alina auf taube Ohren und Unverständnis ihrer Eltern stößt, wenn sie Fragen nach ihrem Großvater stellt. Vielmehr ist es die Wahrnehmung der Konflikte und Diskrepanzen, die sie in ihrer Familie und in ihrem Umfeld beobachtet - „Ihre Rede war so durchsetzt von „Sch-sch!“ und „Psst!“, daß ich zeitweise glaubte, sie litten an einem kollektiven Sprachfehler.“ (S. 24). Dadurch wachsen Alinas Erkenntnis und Mut, Fragen stellen zu müssen. Ihre Mutter war aber nicht bereit, das Schweigen $\mathrm{zu}$ brechen: „Wer keine Antworten bekommt, dem versiegen irgendwann die Fragen, meinte sie.“ (S. 123).

Die Ich-Erzählerin bekam ihre Antworten vom Großvater. Dass er nach anfänglichem Sträuben einwilligte, der Enkelin seine Geschichte zu erzählen, ist angesichts der praktizierten Tabuisierung keine Selbstverständlichkeit und bedarf einer Erläuterung. Aus zwei Gründen offenbart er der Ich-Erzählerin seine Geschichte. Zum einen ist es seine angeschlagene Gesundheit und die Vorahnung des baldigen Todes - am Ende des 
Romans stirbt der Großvater. Ähnlich wie Großmutter Charlotte in Makines „Das französische Testament" dem Ich-Erzähler vor ihrem Tod in einem Brief die Wahrheit seiner Herkunft und die Geschichte ihres Lebens offenbart, so entscheidet der Großvater, sein ganzes Leben Alina zu erzählen: „Du sollst ein Bild bekommen, keine Schnipsel.“ (S. 144). Dafür bleibt den beiden nicht viel Zeit: mit der langersehnten Ausreiserlaubnis wird jeden Tag gerechnet.

Der Vater der Ich-Erzählerin will die Sowjetunion auf jeden Fall vor dem 18. Geburtstag seines Sohnes Willi verlassen. Die seit 20 Jahren erfolglos gestellten Ausreiseanträge werden nicht mehr geduldet: die Familie verkauft ihr Haus, nimmt materielle Verluste in Kauf, zieht um, macht alles, damit die Ausreise vor der Deadline - dem 18. Geburtstag des Sohnes - eingehalten werden kann. Warum wird dieses Datum zu so einem bedeutungstragenden Stichtag? Mit 18 Jahren wird Willi wehrdienstpflichtig und sein Vater hat Angst, dass sein Sohn als Soldat im Afghanistankrieg fallen wird: „Ich will nicht, daß mein Sohn in diesen Krieg zieht.“ (S. 29), „Ich gebe unseren Sohn nicht als Kanonenfutter her." (S. 30). Dass die zehn Jahre andauernde Sowjetische Intervention in Afghanistan (1979-1989) das Leben Tausender Jugendlicher forderte, dass Tausende junger Männer von diesem Krieg physisch und psychisch kaputt heimkehrten, dass Tausende Eltern die sterblichen Reste ihrer Söhne nie zu sehen bekamen, dass Millionen Mütter und Väter in der ganzen Sowjetunion bangen mussten, dass ihr Sohn nach "Afghan“ kommt, ist in der deutschsprachigen Literatur kein Thema. Eleonora Hummel ist neben Vertlib ${ }^{30}$ die erste Autorin, die diese geschichtliche Tatsache in ihrem Roman verortet.

Da der Großvater also weiß, dass die Ausreise der Familie bevorsteht, und er aufgrund seiner Lebenserfahrung weiß, dass es für Alina nicht gut ist, wenn die unbeantworteten Fragen zu einem Konflikt ausufern, der ihr das Ankommen in der Fremde schwer machen könnte, steht er ihr Rede und Antwort: „Niemand fragte danach, weil die Antwort gefürchtet war, und keiner antwortete, weil die Frage ungestellt blieb. Ich weiß auch heute nicht, was einfacher ist, zu schweigen oder zu reden..." (S. 83). Am Ende des Romans scheint der Großvater selbst die ungestellten Fragen bereitwillig zu beantworten:

Wie es weiterging, fragst du, Wnutschka? Ich höre deine Frage nicht, ich sehe sie in deinen Augen. Bei meiner Entlassung habe ich wie viele andere eine Schweigeverpflichtung vorgelegt bekommen. Ich unterschrieb sie, wie so viele andere Papiere zuvor, danach war es vergessen, es hatte keine Bedeutung. Und dennoch, drei Jahrzehnte lang habe ich mich daran gehalten, weil niemand Fragen gestellt hat. Niemand wollte etwas wissen. (S. 214-215).

Dieses Nicht-Wissen-Wollen spiegelt sich metaphorisch in der Angst des Großvaters vor der Dunkelheit wider: „Du lachst mich sicher aus, wenn ich sage, ein alter Mann wie ich hat Angst vor der Dunkelheit“ (S. 55). Wenn der Großvater schweigt, kann er damit rechnen, dass seine Geschichte in Vergessenheit gerät, dass niemand sich an ihn erinnern wird. Neben Dunkelheit werden zwei weitere Symbole verwendet: der Fisch und das Messer. Der Fisch als Symbol ist in diesem Roman sehr präsent, allerdings nicht in der aus dem Christentum bekannten Bedeutung, sondern als ein sich im Schwarm der Artgenossen auflösender Fisch, ein Opfer, das den Netzen des Fischers nicht entkommen wird. Das Taschenmesser, das im Roman zum Vehikel für das Erzählen der Lebensgeschichte des Großvaters wird, kann in diesem Zusammenhang auch 
metaphorisch verstanden werden: es steht für das Brechen des Tabus, aber auch für die Trennung der Familie am Ende des Romans.

\section{Schlussbetrachtungen} Erzählsträngen: die Ich-Erzählerin berichtet über ihr Umfeld - Schule, Sonntagsbesuche bei den Großeltern, den Alltag der Familie - und die Vergangenheit ihres Großvaters. Während der Erzählstrang der Gegenwart hauptsächlich aus Beschreibungen und Kommentaren der Ich-Erzählerin besteht, ist der Erzählstrang der Vergangenheit als Gespräch zwischen dem Großvater und der Enkelin konzipiert. Diese Passagen sind als Dialoge im Roman wiedergegeben, wodurch der bereits angeklungene Gedanke der dialogischen Sprache auch auf die Erzählebene bezogen werden kann. Der Großvater ist der Träger des Wissens, das er an seine Enkelin weitergibt. Er ist nicht nur Gesprächspartner, sondern ein wissender Zeuge, was ihn legitimiert, selbst als IchErzähler aufzutreten.

Während der Großvater aus seiner Vergangenheit berichtet, verwendet er Wörter, die aufgrund seiner Erlebnisse geprägt und kodifiziert sind - „parole vissute“. Indem Eleonora Hummel einen deutschsprachigen Roman schreibt, trägt sie die beschriebenen Ereignisse in ein anderes sprachlich-kulturelles System hinüber. "Gelebte Worte" werden dabei in die deutsche Sprache ihres Romans übernommen, wodurch nicht nur die thematische Erweiterung der deutschsprachigen Literatur erfolgt, sondern die dem deutschen kulturhistorischen Gedächtnis unbekannten Inhalte durch russische Wörter bzw. ihre Übersetzung in der deutschen Literatursprache verortet werden. Wörter wie der schwarze Rabe, Säuberungen, die Bande, das Brudergrab werden zu Verdichtungen des anderen kulturhistorischen Gedächtnisses, das in die deutsche Sprache eingeführt wird. Sie stellen eine neue Form der Präsenz der Erstkultur in der Zweitkultur sowie des kreativen Umgangs mit kulturund sprachimmanenten Besonderheiten dar. Diese sind notwendige Voraussetzungen für das Verständnis der interkulturellen Literatur.

Die Konstellation, dass der wissende Zeuge im Roman auch als Ich-Erzähler auftritt, ist in der interkulturellen Literatur äußerst selten. Geläufig sind das Ausbleiben der Großelterngeneration, der Versuch der Enkelgeneration, die Familiengeschichte selbständig zu verfolgen und die Notwendigkeit, das Familiengeheimnis akzeptieren zu müssen. Dass Eleonora Hummel den Großvater, der nicht der leibliche Großvater der Ich-Erzählerin ist, erzählen lässt, kann dadurch erklärt werden, dass mit der Offenbarung seiner Geschichte die Enttabuisierung ansetzt, die in der Sprache erfolgt. Als Autorin führt sie diesen Enttabuisierungsprozess fort, indem sie die in der russischen Sprache stattgefundenen Ereignisse zu einem deutschsprachigen Roman gerinnen lässt. Die Biografik der beschriebenen Ereignisse wird ihrerseits zum Legitimationsmechanismus für den Sprachwechsel: Da die russische Sprache tabuisiert ist, kann sie nicht als Instanz, die über ästhetische Qualität und Wahrhaftigkeit der Inhalte im Werk wacht, fungieren. Diese Funktion kann die neue, die deutsche Sprache übernehmen, aber erst, wenn der Schriftsteller bzw. der Protagonist sein kulturhistorisches Gedächtnis der neuen Sprache anvertraut hat. Dies erfolgt, indem eine vier Generationen und über 125 Jahre umfassende Geschichte, eine VierGenerationen-Biografie erzählt wird. Somit erweist sich das Schreiben eines 
autobiografischen Romans für den Sprachwechsler als „eine existentielle Notwendigkeit" (Chiellino). Die Besonderheit des Romans Die Fische von Berlin als interkultureller Roman besteht darin, dass er neben den biografischen Zügen die Tendenz zum historischen bzw. dokumentarischen Roman aufweist.

Die dem deutschen Leser unbekannten Ereignisse, Schauplätze und Themen, die das Leben der Menschen in der Sowjetunion insbesondere in den 30er bis 50er Jahren prägten, werden am Beispiel einer Familie über vier Generationen hinweg vorgeführt. Projiziert auf diese Familie, in erster Linie auf das Schicksal des Großvaters der IchErzählerin, lässt die Autorin in Gesprächen zwischen der Enkelin und dem Großvater das faktische Geschehen mit dokumentarischer Genauigkeit wie Kapitel aus der Geschichte der Sowjetunion 1917-1991 folgen. Die aus der Perspektive der russlanddeutschen Aussiedler erzählte Lebensgeschichte ist ein in der deutschsprachigen Literatur kaum etablierter Topos.

Die Großen Säuberungen als Höhepunkt der stalinistischen Diktatur können in der Literatur als Darstellungsform für die absolute Äußerung der Monokultur aufgefasst werden. Gewaltanwendung und Repressalien gegen eigene Bürger sowie Hetzjagd und „Liquidierung“ der s.g. Volksfeinde veranschaulichen, dass der Sowjetstaat bereits einige Jahre nach seiner Gründung in sich implodiert war.

\section{NOTES}

1. Eleonora Hummel, Die Fische von Berlin, Göttingen, Steidl, 2005, S. 151. Wenn nicht anders angegeben, wird weiter aus diesem Roman nur mit Seitenangabe zitiert.

2. Einen aufschlussreichen Sonderfall stellt Natascha Wodin dar, die als Kind ukrainischrussischer Zwangsarbeiter 1945 in einem Arbeitslager auf die Welt kam und in Deutschland aufwuchs. Die mit vielen Preisen ausgezeichnete Autorin fand sehr früh ihre Heimat in der deutschen Sprache. Die 1981 in Odessa geborene Marjana Gaponenko lebt in Mainz und entschied sich 1996 für Deutsch als Sprache ihrer Werke.

3. Weertje Willms spricht von „vier großen Wellen“ in Bezug auf die Emigration aus Russland. Vgl. Weertje Willms: „Wenn ich die Wahl zwischen zwei Stühlen habe, nehme ich das Nagelbrett'. Die Familie in literarischen Texten russischer MigrantInnen und ihrer Nachfahren." In: Michaela Holdenried / Weertje Willms (Hg.): Die interkulturelle Familie. Literatur und sozialwissenschaftliche Perspektiven, Bielefeld, transcript 2012, S. 121-141, hier S. 122-123 (Fußnote 5).

4. Für diesen Roman erhielt Eleonora Hummel 2006 den Adelbert-von-Chamisso-Förderpreis der Robert Bosch Stiftung.

5. Gino Chiellino: Ich in Dresden. Eine Poetikdozentur, Dresden, Thelem 2003, S. 37. Unter dem Namen Gino Chiellino erscheinen Chiellinos Lyrikbände, während er unter dem Namen Carmine Chiellino als Literaturwissenschaftler hervortritt.

6. Vgl. Zur Person: http://www.eleonora-hummel.de/?q=node/48 Zuletzt aufgerufen am 8.09.2012.

7. Vgl. Lena Goreliks Romane „Meine weißen Nächte“ und „Hochzeit in Jerusalem“.

8. Vgl. Julya Rabinowichs Roman „Spaltkopf“... 
9. Der Roman „Das besondere Gedächtnis der Rosa Masur“ von Vladimir Vertlib, der als Gegenbeispiel gelten könnte, entpuppt sich bei genauer Betrachtung als konstruierte Erinnerung der Hauptprotagonistin zwecks kommerzieller Vermarktung.

10. Andreï Makine: Das französische Testament, München, Diana 1999. Weiter wird die Abkürzung "Makine" verwendet.

11. In Anlehnung an Michel Foucault, vgl. dazu Johannes Kabatek: Zur Historizität von Texten, http://www.uni-tuebingen.de/kabatek/discurso/histor.pdf. Zuletzt aufgerufen am 15.09.2012.

12. Zum Begriff "Geschichtstransformationen“ vgl. http:// www.historische.kulturwissenschaft.uni-mainz.de/756.php Zuletzt aufgerufen am 15.09.2012.

13. Vgl. Deutsche Kolonien in Russland und Ukraine: http://www.nexusboard.net/sitemap/6365/ deutsche-kolonien-in-russland-und-ukraine-t296545/ Zuletzt aufgerufen am 9.09. 2012.

14. Vgl. Ebd.

15. Neueren Forschungen zufolge verhungerten 1932/33 fünf bis sieben Millionen Menschen. Nehmen wir die in der Verbannung umgekommenen »Kulaken« und all die anderen Opfer des Kollektivierungs- und Requisitionsterrors hinzu, dann steigt die Schätzung bis auf 14,5 Millionen. Vgl. Stille Vernichtung: http://www.zeit.de/2008/48/A-Holodomor Zuletzt aufgerufen am 9.09.2012.

16. Die Kollektivierung: http://www.bauernhilfe-russland.de/bauer/kollektivierung.htm Zuletzt aufgerufen am 15.09.2012.

17. Vgl. Ludwig Arnold (Hg.): Oskar Pastior, München, edition text \& kritik 2010.

18. Vgl. http://wwii-soldat.narod.ru/OPER/ARTICLES/034-visla.htm Zuletzt aufgerufen am 13.09.12.

19. Severnaja Ėnciklopedija, Bd. 3.5. XX Jhd., Neuzeit, „Norillag“: http://www.emaproject.com/ north_article.html? q=\%D0\%9D\%D0\%BE\%D1\%80\%D0\%B8\%D0\%BB\%D1\%8C\%D0\%BB\%D0\%B0\%D0\%B3 Zuletzt aufgerufen am 11.09.2012. Aus der Statistik der Häftlinge: 1936 - 1251; 1937 - 9139, 1940 - 19 500, 1941 - 20 535, 1945 - 31 822, 1947 - 37 443, 1949 - 57 463, 1951 - 72 490, 1954 - 36 734, 1956 - 13 269 , ebd.

20. Vgl. http://www.solovki.ca/gulag_solovki/20_01.php Zuletzt aufgerufen am 12.09.2012.

21. Vgl. Manfred Hildermeier: Geschichte der Sowjetunion 1917-1991. Entstehung und Niedergang des ersten sozialistischen Staates, München, C. H. Beck 1998, S. 474f, 460-464, 694.

22. Das Lied „Tschornyy woron“, gesungen von einem Kosakenchor, http://www.youtube.com/ watch?v=e_rZgkg2cUA Zuletzt abgerufen am 10.09.2012. Das ukrainische Volkslied heißt: „Oj na hori čorij voron krjače" (Ein schwarzer Rabe kräht auf dem Berg).

23. In vielen slawischen Sprichwörtern ist der Rabe ein Unheilsbringer. Laut Überlieferung wurde er vom Teufel geschaffen, deshalb wird er "schwarz“ in der Bedeutung von „böse“ genannt. Man glaubt, wenn der Rabe sich auf ein Hausdach oder im Hof niederlässt, zeigt er dem Tod sein Opfer. Das Motiv des Raben - „Nur die unsichtbare Schar der Raben / Hör ich mir zu Häupten hungrig schreien.“ (Alfred Margul-Sperber) - und der Krähe - „Fett waren die Krähen, / Die über uns kreisten, / Vom Fleisch unsrer Brüder und Schwestern“ (Alfred Kittner) - findet sich in der Lyrik der deutsch-jüdischen Autoren aus der Bukowina und wird als Metapher für den Tod gebraucht. Vgl. Natalia Shchyhlevska: „Vogelgrenzen in der Bukowiner Dichtung“. In: Frank Hoffmann (Hg.): Grenzüberwindungen - Unüberwindliche Grenzen, Berlin, Lit 2007, S. 247-267. Hingewiesen sei an dieser Stelle auch auf das Lied „U vina dostoinstva, goborjat, celebnije“ (Man sagt, der Wein hätte heilende Wirkung) von Wladimir Wysozkij aus dem Jahr 1967, in dem in der vorletzten Strophe „der schwarze Rabe“ für das Fahrzeug der Polizei verwendet wird.

24. Eigentlich: Cholodetz.

25. Weitere Beispiele für „parole vissute“ wären: „Bande“, „Säuberungen“, „Brudergrab“ sowie „Sauerkraut" und „Salzheringe“, die Alinas Großvater nicht aß, um nicht an seine Lagerzeit erinnert zu werden. 
26. Gino Chiellino (Hg.): Als Dichter in Deutschland, Dresden, Thelem 2011, S. 16-18.

27. Ebd., S. 16.

28. a.a.O. Gino Chiellino: Ich in Dresden. Eine Poetikdozentur, S. 50.

29. a.a.O. Gino Chiellino (Hg.): Als Dichter in Deutschland, S. 16.

30. Vladimir Vertlib: Zwischenstationen, München, Deutscher Taschenbuch Verlag 2005, S. 242: „Als wir die Sowjetunion verlassen haben, dachten wir uns: Gott sei Dank [...] Wir haben ihm Afghanistan erspart."

\section{RÉSUMÉS}

La présente contribution se concentre, à partir de l'étude du roman Die Fische von Berlin [Les poissons de Berlin] de Eleonora Hummel, sur les aspects historiques et politiques ainsi que sur les expériences personnelles de la persécution et de l'émigration en tant que thèmes centraux de la littérature germano-russe. Elle analyse la manière dont les nouveaux thèmes et événements, que le lecteur allemand ne connaît pas encore, sont intégrés dans la structure narrative, puis interprétés. Dans ce contexte, la mémoire joue un rôle prépondérant. On verra que la littérature germano-russe élargit non seulement le spectre des thèmes et motifs de la littérature contemporaine allemande tout en comblant des lacunes dans la mémoire collective, mais qu'elle permet également un nouveau regard sur l'histoire de l'Union soviétique. On abordera en outre le maniement de la langue allemande en tant que langage littéraire, afin d'illustrer de quelle manière l'expérience de la transgression est mise en parole.

Taking the novel Die Fische von Berlin as its focus, this paper discusses the specific historical and political points of reference and the personal experiences of persecution and emigration that constitute the central themes of Russian-German literature (russlanddeutsche Literatur). It analyses how new settings, themes and events largely unfamiliar to the German reader are built in to and framed by the novel's narrative structure, with remembrance occupying a position of particular importance. The paper argues that Russian-German literature not only expands contemporary German literature's spectrum of themes and motifs, filling the gaps in collective memory, but also casts a new light on the history of the Soviet Union. The paper also examines how German literary language is used in this text to illustrate how border-crossing experiences find stylistic expression.

Der vorliegende Beitrag fokussiert am Beispiel des Romans Die Fische von Berlin die geschichtlichpolitischen Bezugspunkte und die persönlichen Erfahrungen von Verfolgung und Emigration als zentrale Themen der russlanddeutschen Literatur. Er analysiert, wie neue, dem deutschen Leser unbekannte Schauplätze, Themen und Ereignisse in die Erzählstruktur eingebaut und dargestellt werden. In diesem Kontext kommt dem Erinnern eine besondere Bedeutung zu. Es wird aufgezeigt, dass die Literatur der Russlanddeutschen nicht nur das thematische und motivische Spektrum der deutschen Gegenwartsliteratur erweitert und Lücken im kollektiven Gedächtnis füllt, sondern einen neuen Blick auf die Geschichte der Sowjetunion ermöglicht. Darüber hinaus wird auf die Handhabung der deutschen Sprache als Literatursprache eingegangen, um zu veranschaulichen, wie die Erfahrung der Grenzüberschreitung versprachlicht wurde. 


\section{AUTEURS}

\section{NATALIA SHCHYHLEVSKA}

Universität Mainz 